Document downloaded from:

http://hdl.handle.net/10251/60873

This paper must be cited as:

Oliverio, F.; Nuin Pla, NE.; Andreu Ros, MI.; Ragno, G.; Miranda Alonso, MÁ. (2014). Assessment of drug entrapment within liposomes using photophysical probes. European Journal of Pharmaceutics and Biopharmaceutics. 88(2):551-555. doi:10.1016/j.ejpb.2014.06.013.

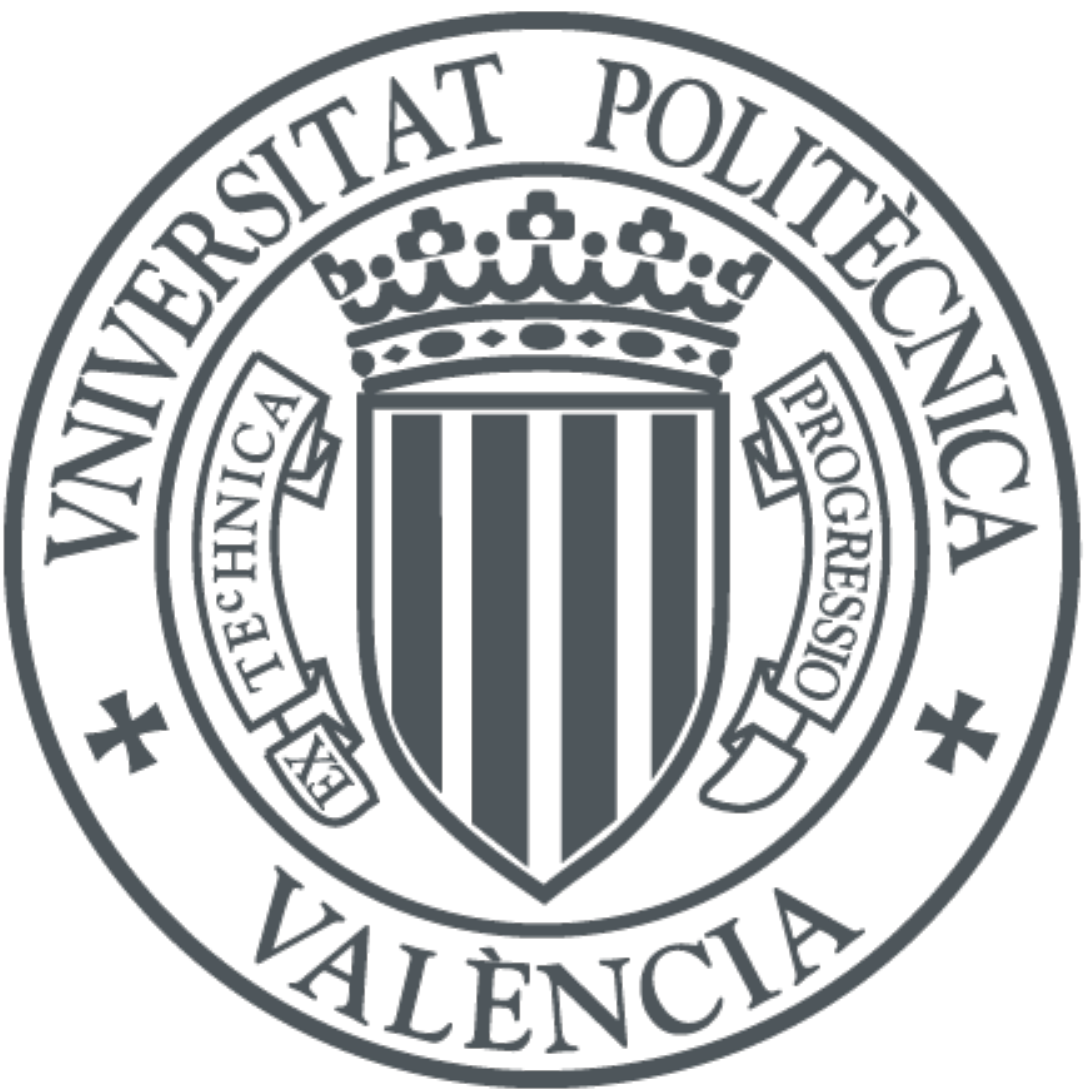

The final publication is available at

http://dx.doi.org/10.1016/j.ejpb.2014.06.013

Copyright Elsevier

Additional Information 


\section{Assessment of drug entrapment within liposomes using}

\section{photophysical probes}

Filomena Oliverio, ${ }^{a, b}$ Edurne Nuin, ${ }^{a}$ Inmaculada Andreu, ${ }^{c}$ Gaetano Ragno, ${ }^{b, *}$ and Miguel A. Miranda ${ }^{a^{*}}$

anstituto de Tecnología Química (UPV-CSIC) Universidad Politécnica de Valencia. Consejo Superior de Investigaciones Científicas. Avenida de los Naranjos s/n. 46022 Valencia. Spain

${ }^{\mathrm{b}}$ Department of Pharmacy, Health and Nutritional Sciences, University of Calabria, 87036 Rende (CS), Italy

${ }^{c}$ Unidad Mixta de Investigación IIS La Fe-UPV, Hospital La Fe, Bulevar Sur s/n. 46026 Valencia, Spain

RECEIVED DATE (to be automatically inserted after your manuscript is accepted if required according to the journal that you are submitting your paper to)

CORRESPONDING AUTHOR FOOTNOTE

Prof. Miguel A. Miranda

E-mail: mmiranda@qim.upv.es

Prof. Gaetano Ragno

E-mail: gaetano.ragno@unical.it 


\section{Abbreviations:}

CIN: $(R)$-cinacalcet

CryoSEM: Cryoscopic Scanning Electronic Microscopy

DLS: Dynamic Light Scattering

EE: Encapsulation efficiency

$\mathrm{E}_{\mathrm{s}}$ : Singlet energy

HPLC: High performance liquid chromatography

$\mathrm{k}_{\mathrm{Fq}}$ : Fluorescence quenching rate constant for oxygen

$\mathrm{k}_{\mathrm{Tq}}$ : Triplet quenching rate constant for oxygen

Lip: Liposome

LFP: Laser flash photolysis

LUV: Large unilamellar liposomes

NPX: $(S)$-naproxen

PBS: Phosphate buffered saline solution

PC: 1,2-dioleoyl-sn-glycero-3-phosphocholine

PTI: Photon Technology International

TEM: Transmission Electronic Microscopy

TLE: Thin layer evaporation

$\tau_{\mathrm{s}}$ : Singlet lifetime

$\tau_{\mathrm{T}}$ : Triplet lifetime

UV-Vis: Ultraviolet-visible 


\begin{abstract}
The photophysical and photochemical behavior of $(R)$-cinacalcet $(\mathrm{CIN})$ and $(S)$-naproxen (NPX) entrapped within liposomes has been studied. For this purpose, liposome encapsulated drugs have been prepared through thin layer evaporation and characterized by transmission electron microscopy, cryoscopy scanning electron microscopy and dynamic light scattering. Steady state and time-resolved fluorescence experiments showed similar spectra, emission quantum yields, singlet energies and lifetimes for the selected drugs, outside and inside liposomes. By contrast, laser flash photolysis experiments revealed an important enhancement of the triplet lifetimes for entrapped drugs inside liposomes, indicating the spatial confinement existing in the microenvironment prevailing in these biomimetic entities. Thus, this photophysical property shows potential as a non-invasive, direct and valuable tool to monitor encapsulation of photoactive drugs and to probe the intraliposome environment. In addition, it provides a new quantitative indicator of the capability of liposomes to act as drug carriers.
\end{abstract}

Keywords: Cinacalcet, Drug Carrier, Drug Delivery, Fluorescence, Laser Flash Photolysis, Liposome, Naphthalene Chromophore, Naproxen, Singlet Excited states, Triplet Excited States. 


\section{Introduction}

Liposomes are the smallest artificial vesicles of spherical shape that can be produced from natural phospholipids and cholesterol. They are very versatile tools in biology, biochemistry and medicine $[1,2,3,4,5]$ and have attracted attention as potential vehicles for drug delivery to selected cells or tissues in vivo and as carriers of drugs or other bioactive compounds including proteins, hormones and diagnostic agents.[6,7] Although the interest has been mainly focused on drug delivery, a variety of other applications have been found in cosmetics, cleansing and food technology.[6,8]

Liposomes are simple biomimetic models for cell membranes and provide appropriate microenvironment for the study of photoinduced electron transfer[9] and lipid peroxidation.[10,11] Adoption of this matrix seems very promising in photodynamic therapy, based on the generation of reactive oxygen species that destroy the tissues or target cells by the use of photosensitizing compounds, which absorb photons and are excited to a triplet state, capable of generating superoxide anion or promoting molecular oxygen to its highly reactive singlet state. This mechanism seems responsible for cell death in tumor tissues or for stimulation of immune responses that cause cell death. Despite the progress made in the study of clinically available photosensitizing agents, there are still several drawbacks limiting their use. For example, their hydrophobic nature limits their efficiency and delivery in the body. To circumvent these problems, alternative formulations are required to enhance solubility and to achieve selective delivery to tumor sites. In this context, liposomes have the ability to encapsulate hydrophobic molecules. Inclusion of photosensitizers within this type of matrix has revealed a good correlation between the physicochemical features of formulations and their photosensitizing efficiency.[12] Moreover, interaction of light with photosensitive liposomes may cause membrane reorganization and offers an appropriate tool for selective drug release in the target site, with a significant impact on the therapeutic index of drugs. Additional applications of this effect can be found in imaging or sensing.[13] The study of molecular mechanisms that result in photo-destabilization of liposome membranes is essential in developing light-activable liposomes for photodynamic therapy with higher opportunities of success in the clinical practice.[14] 
Recently, naphthalene-like triplet excited states have been used as reporters for the interaction of drugs with transport proteins[15,16,17] and for the incorporation of photoactive compounds within cholic acid aggregates[18] and mixed micelles.[19] In the present work, two naphthalene-derived drugs, namely $(R)$-Cinacalcet (CIN) and (S)-Naproxen (NPX) have been entrapped within liposomes, and photophysical studies have been performed in order to establish how this microenvironment influences the excited state dynamics of both drugs. The chemical structures of CIN and NPX are reported in Chart1. The former (CIN) is a recently marketed calcimimetic drug, mimicking the action of calcium on tissues, by allosteric activation of the calcium-sensing receptor.[20,21] This agent is used for treatment of persistent hyperparathyroidism in renal transplanted patients, with chronic kidney disease and renal failure in dialysis.[22,23,24] It is also used for the treatment of hypercalcemia in patients with parathyroid carcinoma, in order to decrease the calcium level in blood.[25,26,27] As regards NPX it is widely used as a highly effective nonsteroidal anti-inflammatory drug. Although NPX is rather safe and tolerable, serious gastrointestinal side effects, and in some cases skin photosensitivity, may appear after oral administration.[28,29,30,31] Besides, it exhibits red blood cells photohaemolytic activity and causes photocleavage of DNA.[28,29,32]

At the present stage, our goal is to assess the entrapment of both naphthalene-derived drugs within biomimetic liposomes by means of non-invasive and straightforward spectroscopic tools, specifically fluorescence and laser flash photolysis (LFP). Selection of CIN and NPX as probes seems appropriate, since they present a two-channel responsive chromophore and appear in principle suitable systems to evaluate their encapsulation inside liposomes by photophysical techniques.

\section{Materials and Methods}

Materials. NPX and 1,2-dioleoyl-sn-glycero-3-phosphocholine (PC), with purity higher than $99 \%$, were purchased from Sigma-Aldrich (Steinheim, Germany). The latter was stored as purchased at $20^{\circ} \mathrm{C}$. CIN was extracted from commercial Mimpara $60 \mathrm{mg}$ (Amgen, Spain). The content of six pills was powdered in a mortar, suspended in $\mathrm{NH}_{4} \mathrm{OH} 1 \mathrm{M}(50 \mathrm{~mL})$ and extracted with $\mathrm{CH}_{2} \mathrm{Cl}_{2}(4 \mathrm{x} 50 \mathrm{~mL})$. 
The combined organic layers were washed with brine $(3 \times 150 \mathrm{~mL})$ and water $(3 \times 150 \mathrm{~mL})$, dried over $\mathrm{MgSO}_{4}$, and evaporated under reduced pressure. The residue was purified by column chromatography through silica gel 60 (dichloromethane:methanol:ammonium hydroxide 98:2:0.1 v/v/v) to give CIN as a colorless oil. The purity of isolated CIN was higher than $98 \%$ as indicated by ${ }^{1} \mathrm{H}$ and ${ }^{13} \mathrm{C}$ NMR spectroscopy. Chloroform and methanol were of HPLC grade from Scharlab (Sentmenat, Spain) and were used without further purification. Phosphate buffered saline solution (PBS) $(\mathrm{pH}=7.4,0.01 \mathrm{M})$ was prepared by dissolving Sigma tablets in the appropriate amount of purified water (Milli-Q system, Millipore).

Preparation of liposomes. Liposomes including CIN and NPX were prepared by thin layer evaporation (TLE) method according to standard conditions.[33,34] Liposomes were obtained dissolving $12.1 \mathrm{mg}$ of $\mathrm{PC}$ in $10 \mathrm{~mL}$ of chloroform containing the drug $\left(\mathrm{ca} .2 \times 10^{-3} \mathrm{M}\right)$ in a $50 \mathrm{~mL}$ roundbottomed flask. Drug-free liposomes were prepared as blank. The solutions were evaporated at $58{ }^{\circ} \mathrm{C}$ by using a vacuum rotary evaporator for $10 \mathrm{~min}$, to remove the organic solvents. Then, the residual solvent was completely eliminated by drying under vacuum for 2 hours. The obtained lipid film was hydrated with $2 \mathrm{~mL}$ of PBS, leading to a liposomal formulation. Large unilamellar liposomes (LUV) were produced starting from multilamellar ones by sonication in an ultrasonic bath for $30 \mathrm{~min}$ at $50{ }^{\circ} \mathrm{C}$. In this way, more homogeneous size vesicles were obtained; they were kept under controlled temperature (4 ${ }^{\circ} \mathrm{C}$ ) until their use within 3 days. Immediately before use, $0.5 \mathrm{~mL}$ of liposome emulsion were added to the same amount of PBS and purified by centrifugation at $14000 x \mathrm{~g}$ during $10 \mathrm{~min}$ for 3 times at room temperature in a HERAUS Pico 21 centrifuge (Thermo electron corporation, Germany).

Size analysis. Liposomes were characterized by Transmission Electronic Microscopy (TEM), Cryoscopic Scanning Electronic Microscopy (CryoSEM) and Dynamic Light Scattering (DLS). TEM observation of liposomes was carried out by a JEOL2010-FEG TEM/STEM microscope, equipped with a field emission electron source and an objective lens with Cs value of $0.5 \mathrm{~mm}$. The images were digitally recorded on a 1024 x 1024 CCd camera. Image analysis was performed using routines implemented in the software plugins of Digital Micrograph. For TEM measurements, $5 \mu \mathrm{L}$ of purified 
liposomes were put on a holey-carbon coated 3-mm $\mathrm{Cu}$-grid and dried at room temperature under a cabinet equipped with an aspiration system. After complete evaporation of the solvent, the sample was ready for direct observation. SEM images were obtained using a JEOL JSM-6300 microscope operating at $20 \mathrm{kV}$. For CryoSEM analysis, samples were frozen, and thin frozen sections were imaged without staining. For both microscopic analysis liposomes were rehydrated with Millipore water instead of PBS, in order to avoid the optical interference of saline crystals in the medium. DLS measurements were conducted using a Zetasizer Nano ZS (Malvern Instruments Ltd., Worcestershire, United Kingdom). The liposomes were diluted with deionized water and the experiments were performed at $25{ }^{\circ} \mathrm{C}$ and $173^{\circ}$ scattering angle. The mean hydrodynamic diameter was determined by cumulant analysis.

Drug entrapment efficiency. The encapsulation efficiency (EE) of CIN and NPX was determined using equation $1 ;[35]$ it is expressed as the percentage of the drug trapped in purified liposomes referred to the non purified solutions.

$$
\text { Encapsulation efficiency }(\%)=\frac{C t-C o}{C t} \times 100
$$

where $C o$ is the concentration in the supernatant diluted with PBS and $C t$ refers to the liposome emulsion diluted with warm ethanol $\left(70^{\circ} \mathrm{C}\right)$, for disruption of the liposomes and release of the included drug to the solvent. The amount of entrapped drug was determined from the absorbance of the samples by UV-Vis spectrophotometry at the maximum $(230 \mathrm{~nm})$. Encapsulation efficiency was verified at each preparation of new batch of liposomes.

Spectroscopic measurements. UV spectra were recorded on a Cary 300 (Varian) spectrophotometer. Steady-state fluorescence experiments were carried out using a Photon Technology International (PTI, Germany) LPS-220B spectrofluorometer, equipped with a monochromator in the wavelength range of 200-700 nm. The time-resolved fluorescence determinations were performed with a Time Master fluorescence lifetime spectrometer TM 2/2003 from PTI, using a hydrogen/nitrogen flash lamp as the excitation source. The kinetic traces were fitted by exponential functions, with using a reconvolution procedure to separate from lamp pulse profile. For fluorescence measurements, concentrations were 
fixed by adjusting the absorbance for CIN and NPX solutions at the arbitrary value of 0.1 at the excitation wavelength of $290 \mathrm{~nm}$ and $330 \mathrm{~nm}$, respectively. Fluorescent quantum yield measurements were obtained with nitrogen-bubbled PBS solutions; the absorbance was adjusted at the excitation wavelength using $\operatorname{NPX}\left(\phi_{\mathrm{f}}=0.47\right.$ in acetonitrile $)[28]$ and 1-methylnaphthalene $\left(\phi_{\mathrm{f}}=0.19\right.$ in acetonitrile)[36] as standards. All experiments were conducted at room temperature using $10 \times 10 \mathrm{~mm}^{2}$ quartz cells with $4 \mathrm{~mL}$ capacity and were run in triplicate.

Laser flash photolysis. Laser flash photolysis (LFP) experiments were performed by using a Qswitched Nd:YAG laser (Quantel Brilliant, $266 \mathrm{~nm}, 14 \mathrm{~mJ}$ pulse $^{-1}, 5 \mathrm{~ns}$ fwhm) coupled to a mLFP-111 Luzchem miniaturized equipment. In general, solutions were nitrogen-purged for 10 min before acquisition, and the triplet decays were registered at $420 \mathrm{~nm}$. Samples were prepared in PBS, and the absorbance was kept at ca. 0.3. Solutions of CIN and NPX in PBS, treated in the same way as liposomes, were used as reference. All LFP analyses were performed at room temperature using 10 x 10 $\mathrm{mm}^{2}$ quartz cells with $4 \mathrm{~mL}$ capacity and were run in triplicate.

\section{Results and Discussion}

Photophysical studies were performed on CIN and NPX entrapped within liposomes, in order to establish how this microenvironment influences the excited state dynamics of the drugs.

Entrapment of drugs within liposomes. Large unilamellar liposomes (LUV) have been reported since 1965 as membrane biomimetics.[37,38] In this work, optimization of the classical TLE method was carried out in an attempt to increase the encapsulation efficiency (EE) and to obtain liposomes with homogeneous size. Thus, the EE were $c a .40 \%$ in all cases. Moreover, this parameter appeared to be very sensitive to the storage of liposomes. In fact, whereas the preparations were stable at $4^{\circ} \mathrm{C}$ for at least 3 days, maintenance at room temperature caused a progressive decrease of EE, which dropped to $15 \%$ after 2 days, with an important transfer of free drug from liposomes to the PBS solution.

The obtained solutions were characterized using DLS, TEM and CryoSEM microscopy (Figure 1). The diameter of prepared liposomes was determined using DLS measurements. This size was found in 
the range of 80-260 $\mathrm{nm}$ (with a maximum at $130 \mathrm{~nm}$ and PDI $c a .0 .3$ ) corresponding to LUV, which makes them appropriate as biomimetic models. Both microscopy techniques are also suitable to investigate the size and external features of the liposomes. In particular, TEM analysis is widely used in this field[39] because it can offer an unrefined image of the liposomal profile and permits size measurements. On the other hand, CryoSEM analysis shows liposome morphology in the hydrated state: in general, the expected spheroidal shape was observed. Uniform and compact bilayers, a typical mark of unilamellar liposomes, were clearly identified; smaller liposomes were rarely observed (Figure 1A). In some cases, small inclusions on the surface were found, probably lipids from disrupted liposomes during analysis, in contact with the same surface.

\section{Figure 1.}

Photophysics of drugs within liposomes. The emission spectra of CIN and NPX in homogeneous medium and incorporated within liposomes were recorded at room temperature, using $290 \mathrm{~nm}$ and 330 $\mathrm{nm}$ as the excitation wavelengths, respectively (see Figure 2). This selection was done in order to promote the chromophores to their lowest-lying singlet excited states, which usually results in enhanced selectivity and emission efficiency. This way, the bulk concentrations needed to obtain absorbance of $c a$. 0.1 are in the order of $10^{-5} \mathrm{M}$. Solutions of CIN and NPX outside and inside liposomes showed the same spectra, displaying their corresponding maxima at 320 and $355 \mathrm{~nm}$. The lack of a significant bathochromic or hypsochromic shift associated with encapsulation indicates that emission from these photoactive units is not particularly sensitive to the hydrophobic medium provided by the liposomes. The shape and position of the main bands of excitation spectra were essentially coincident with those of the absorption spectra. This confirms that emission takes place from the locally excited singlet and does not involve impurities, ground-state complexes or aggregates. From the intersection between normalized excitation and emission traces, singlet energies $\left(E_{s}\right)$ were obtained for all systems (Table 1). The values indicate little perturbation if any of the relevant molecular orbital levels. Accordingly, the fluorescence quantum yields inside liposomes for CIN $\left(\phi_{\mathrm{f}}=0.11\right)$ and NPX $\left(\phi_{\mathrm{f}}=0.40\right)$ were also similar to the values 
obtained for the free drugs. In order to detect a possible dynamic quenching, time resolved fluorescence studies were carried out. In general, this type of quenching is detected through a faster decay and reveals a higher reactivity. This was not the case under the experimental conditions, as similar lifetimes were determined for the singlet excited state outside and inside liposomes.

\section{Table 1.}

The rate constant of singlet excited state quenching by oxygen in PBS was also determined using time-resolved fluorescence spectroscopy, recording decays under $\mathrm{N}_{2}$, air, and $\mathrm{O}_{2}$ atmosphere. The lifetimes of CIN and NPX within liposomes were slightly more sensitive to the presence of oxygen than those of the free drugs in solution. This was not unexpected, since both the real oxygen concentrations and the diffusion rate inside liposome should differ from the values found in bulk PBS. From these data, the apparent quenching rate constant by oxygen (assuming similar effective oxygen concentration) was determined by means of the Stern-Volmer relationships and found to be $1.4 \times 10^{10} \mathrm{M}^{-1} \mathrm{~s}^{-1}$ for free CIN and $1.5 \times 10^{10} \mathrm{M}^{-1} \mathrm{~s}^{-1}$ for liposome-encapsulated CIN, $8.8 \times 10^{9} \mathrm{M}^{-1} \mathrm{~s}^{-1}$ for free NPX, $9.6 \times 10^{9} \mathrm{M}^{-1} \mathrm{~s}^{-1}$ for liposome-encapsulated NPX.

\section{Figure 2.}

In addition to fluorescence measurements, LFP experiments were carried out in order to investigate the influence of liposome encapsulation drugs on the lifetime of the excited states, in the nanosecondmicrosecond timescale, using a Q-switched Nd:YAG laser for excitation at $266 \mathrm{~nm}$, where the absorbance of the samples was $c a .0 .3$ (in the order of $10^{-4} \mathrm{M}$ ). This technique allows detecting longerlived transients, which may move and relocate during their lifetimes, or even establish a dynamic equilibrium between the populations of different compartments. Moreover, the wider dynamic range is more convenient for safe detection of slow processes and for assessment of possible protection of the species of interest from reaction with a variety of reagents, including oxygen or adventitious quenchers. The transient absorption spectra of CIN and NPX in solution exhibited the typical naphthalene-like triplet-triplet absorption band[28] in the $420-450 \mathrm{~nm}$ region that remained unchanged upon 
encapsulation inside liposome (Figure 3). However, it is worth mentioning that the spectra of Figure 3 show the absence of naphthalene-like radical cations, which appear in PBS solution in the longwavelength edge of the selected window, especially in the case of NPX. This is consistent with the hydrophobic nature of the intraliposome region, which prevents photoionization.

Interestingly, enhancements of the triplet lifetimes (beyond $40 \mu \mathrm{s}$ ) were observed for encapsulated drugs, revealing the sensitivity of this excited state to the experienced microenvironment (see Figure 3 and Table 1). In this context, the liposomes can be considered as microheterogeneous systems, an expression commonly used for homogeneous media at the macroscopic level (looking like clear and transparent preparations), where distinct microscopic compartments are defined with different physicochemical properties (hydrophobicity, polarity, viscosity, refraction index, etc.), which results in a significant degree of anisotropy. Further examples of microheterogeneous systems are cyclodextrins,[40] micelles,[19,41] transport proteins,[42] nanoparticles,[43] bile salt aggregates,[18] etc. Again, the apparent triplet quenching rate constants were determined from the triplet lifetimes under $\mathrm{N}_{2}$, air and $\mathrm{O}_{2}$, in the different media. The obtained values were $1.8 \times 10^{9} \mathrm{M}^{-1} \mathrm{~s}^{-1}$ for free $\mathrm{CIN}, 1.2 \times 10^{9} \mathrm{M}^{-1} \mathrm{~s}^{-1}$ for liposome-encapsulated CIN, $2.0 \times 10^{9} \mathrm{M}^{-1} \mathrm{~s}^{-1}$ for free NPX and $9.9 \times 10^{8} \mathrm{M}^{-1} \mathrm{~s}^{-1}$ for liposome-encapsulated NPX. Thus, the medium-dependent decay of the triplet excited states of CIN and NPX, as revealed by the lengthening of the lifetimes, is the most sensitive photophysical parameter to the nature of the microenvironment provided by the interior of liposomes. As a consequence, this property can in principle be used to monitor encapsulation of photoactive drugs and to probe the interior of these biomimetic systems.

\section{Figure 3.}

\section{Conclusions}

The present work has revealed an efficient inclusion of the drugs in the microenvironment provided by liposomes. The most remarkable photophysical property is the increase in the triplet lifetimes of CIN and NPX observed inside liposomes. This agrees well with the spatial confinement existing in the 
interior of such biomimetic systems, where the degrees of freedom and hence the possibilities of nonradiative decay are restricted. Hence, transient absorption spectroscopy is a non-invasive, direct and valuable tool to assess the encapsulation of photoactive drugs inside liposomes and its influence on the excited state dynamics, thus providing a new quantitative indicator of the capability of liposomes to act as drug carriers.

Acknowledgment. We gratefully acknowledge financial support from the Carlos III Institute of Health (Servet Contract CP11/00154 for I.A. and Red RETICS de investigación de Reacciones Adversas a Alergenos y Fármacos, RIRRAF) and from Ministero dell'Istruzione, dell'Università e della Ricerca (M.I.U.R. Italy)

\section{References}

[1] P. Chetanachan, P. Akarachalanon, D. Worawirunwong, P. Dararutana, A. Bangtrakulnonth, M. Bunjop, S. Kongmuang, Ultrastructural characterization of liposomes using transmission electron microscopy, in: T. Tunkasiri (Ed.), Smart Materials, Trans Tech Publications Ltd, StafaZurich, 2008, pp. 709-711.

[2] D. D. Lasic, D. Papahadjopoulos, Liposomes revisited, Science 267 (1995) 1275-1276.

[3] X. F. Liang, H. J. Wang, H. Luo, H. Tian, B. B. Zhang, L. J. Hao, J. I. Teng, J. Chang, Characterization of novel multifunctional cationic polymeric liposomes formed from octadecyl quaternized carboxymethyl chitosan/cholesterol and drug encapsulation, Langmuir 24 (2008) 7147-7153.

[4] W. J. M. Mulder, G. J. Strijkers, G. A. F. Van Tilborg, A. W. Griffioen, K. Nicolay, Lipid-based nanoparticles for contrast-enhanced MRI and molecular imaging, NMR Biomed. 19 (2006) 142164.

[5] A. Sharma, U. S. Sharma, Liposomes in drug delivery: progress and limitations, Int. J. Pharm. 154 (1997) 123-140. 
[6] A. K. Greene, Liposomes as drug carriers: recent trends and progress, John Wiley \& Sons, New York, 1988.

[7] D. D. Lasic, In vesicles, Marcel Dekker, New York, 1987.

[8] D. D. Lasic, Novel applications of liposomes, Trends Biotechnol. 16 (1998) 307-321.

[9] B. Armitage, D. F. O'Brien, Vectorial photoinduced electron transfer between phospholipid membrane-bound donors and acceptors, J. Am. Chem. Soc. 114 (1992) 7396-7403.

[10] S. N. Chatterjee, S. Agarwal, Liposomes as membrane model for study of lipid-peroxidation, Free Radical Biol. Med. 4 (1988) 51-72.

[11] L. Xu, T. A. Davis, N. A. Porter, Rate constants for peroxidation of polyunsaturated fatty acids and sterols in solution and in liposomes, J. Am. Chem. Soc. 131 (2009) 13037-13044.

[12] C. Bombelli, G. Caracciolo, P. P. Di, M. Diociaiuti, P. Luciani, G. Mancini, C. Mazzuca, M. Marra, A. Molinari, D. Monti, L. Toccacieli, M. Venanzi, Inclusion of a photosensitizer in liposomes formed by DMPC/gemini surfactant: correlation between physicochemical and biological features of the complexes, J. Med. Chem. 48 (2005) 4882-4891.

[13] D. E. Bennett, H. Lamparski, D. F. O'brien, Photosensitive liposomes, J. Lipid. Res. 4 (1994) 331348.

[14] A. Yavlovich, B. Smith, K. Gupta, R. Blumenthal, A. Puri, Light-sensitive lipid-based nanoparticles for drug delivery: design principles and future considerations for biological applications, Mol. Membr. Biol. 27 (2010) 364-381.

[15] E. Nuin, I. Andreu, M. J. Torres, M. C. Jimenez, M. A. Miranda, Enhanced photosafety of cinacalcet upon complexation with serum albumin, J. Phys. Chem. B 115 (2011) 1158-1164.

[16] E. Nuin, M. C. Jimenez, G. Sastre, I. Andreu, M. A. Miranda, Drug-drug interactions within protein cavities probed by triplet-triplet energy transfer, J. Phys. Chem. Lett. 4 (2013) 1603-1607.

[17] R. Perez-Ruiz, R. Alonso, E. Nuin, I. Andreu, M. C. Jimenez, M. A. Miranda, Naphthalene triplet excited state as a probe for the assessment of drug distribution in binary protein systems, J. Phys. Chem. B 115 (2011) 4460-4468. 
[18] M. Gomez-Mendoza, E. Nuin, I. Andreu, M. L. Marin, M. A. Miranda, Photophysical probes to assess the potential of cholic acid aggregates as drug carriers, J. Phys. Chem. B 116 (2012) 10213-10218.

[19] E. Nuin, M. Gomez-Mendoza, M. L. Marin, I. Andreu, M. A. Miranda, Influence of drug encapsulation within mixed micelles on the excited state dynamics and accessibility to ionic quenchers, J. Phys. Chem. B 117 (2013) 9327-9332.

[20] G. N. Kumar, C. Sproul, L. Poppe, S. Turner, M. Gohdes, H. Ghoborah, D. Padhi, L. Roskos, Metabolism and disposition of calcimimetic agent cinacalcet HCL in humans and animal models, Drug Metab. Dispos. 32 (2004) 1491-1500.

[21] A. Peck, Calcimimetics: a review of the recent literature, Kidney 18 (2009) 175-179.

[22] T. Apostolou, L. Damianou, V. Kotsiev, S. Drakopoulos, V. Hadjiconstantinou, Treatment of severe hypercalcemia due to refractory hyperparathyroidism in renal transplant patients with the calcimimetic agent cinacalcet, Clin. Nephrol. 65 (2006) 374-377.

[23] T. Kawata, Y. Imanishi, K. Kobayashi, N. Onoda, S. Okuno, Y. Takemoto, T. Komo, H. Tahara, M. Wada, N. Nagano, E. Ishimura, T. Miki, T. Ishikawa, M. Inaba, Y. Nishizawa, Direct in vitro evidence of the suppressive effect of cinacalcet $\mathrm{HCl}$ on parathyroid hormone secretion in human parathyroid cells with pathologically reduced calcium-sensing receptor levels, J. Bone Miner. Metab. 24 (2006) 300-306.

[24] A. E. Kruse, U. Eisenberger, F. J. Frey, M. G. Mohaupt, Effect of cinacalcet cessation in renal transplant recipients with persistent hyperparathyroidism, Nephrol. Dial. Transplant. 22 (2007) $2362-2365$.

[25] S. J. Silverberg, M. R. Rubin, C. Faiman, M. Peacock, D. M. Shoback, R. C. Smallridge, L. E. Schwanauer, K. A. Olson, P. Klassen, J. P. Bilezikian, Cinacalcet hydrochloride reduces the serum calcium concentration in inoperable parathyroid carcinoma, J. Clin. Endocrinol. Metab. 92 (2007) 3803-3808. 
[26] E. D. Szmuilowicz, R. D. Utiger, A case of parathyroid carcinoma with hypercalcemia responsive to cinacalcet therapy, Nat. Clin. Pract. Endocrinol. Metab. 2 (2006) 291-296.

[27] S. E. Rodgers, N. D. Perrier, Parathyroid carcinoma, Curr. Opin. Oncol. 18 (2006) 16-22.

[28] F. Bosca, M. L. Marin, M. A. Miranda, Photoreactivity of the nonsteroidal anti-inflammatory 2arylpropionic acids with photosensitizing side effects, Photochem. Photobiol. 74 (2001) 637655.

[29] M. Partyka, B. H. Au, C. H. Evans, Cyclodextrins as phototoxicity inhibitors in drug formulations: studies on model systems involving naproxen and $\beta$-cyclodextrin, J. Photochem. Photobiol. A: Chem. 140 (2001) 67-74.

[30] M. O. Reynier, J. C. Montet, C. Marteau, C. Crotte, A. M. Montet, A. Gerolami, H. Sarles, Intestinal-absorption and micellar solubilization of cholesterol, Gastroenterology 76 (1979) $1225-1225$.

[31] G. Stuttgen, The present status of anti-inflammatory agents in dermatology, Drugs 36 (1988) 43-48.

[32] L. L. Costanzo, G. De Guidi, G. Condorelli, A. Cambria, M. Fama, Molecular mechanism of naproxen photosensitization in red blood cells, J. Photochem. Photobiol. B: Biol. 3 (1989) 223235.

[33] L. Tavano, R. Muzzalupo, S. Trombino, R. Cassano, A. Pingitore, N. Picci, Effect of formulations variables on the in vitro percutaneous permeation of sodium diclofenac from new vesicular systems obtained from pluronic triblock copolymers, Colloids Surf. B-Biointerfaces 79 (2010) 227-234.

[34] S. Watanabe, M. Iwamura, A liposome-forming caged compound: synthesis and photochemical properties of a caged L-leucyl-L-leucine methyl ester with a steroid skeleton, J. Org. Chem. 62 (1997) 8616-8617.

[35] T. Nii, F. Ishii, Encapsulation efficiency of water-soluble and insoluble drugs in liposomes prepared by the microencapsulation vesicle method, Int. J. Pharm. 298 (2005) 198-205. 
[36] S. L. C. Murov, I. G. L. Hug, , Handbook of Photochemistry, 2nd ed., Marcel Dekker, New York, 1993.

[37] A. D. Bangham, M. M. Standish, J. C. Watkins, Diffusion of univalent across lamellae of swollen phospholipids, J. Mol. Biol. 13 (1965) 238-\&.

[38] C. Bernsdorff, A. Wolf, R. Winter, E. Gratton, Effect of hydrostatic pressure on water penetration and rotational dynamics in phospholipid-cholesterol bilayers, Biophys. J. 72 (1997) 1264-1277.

[39] G. De Rosa, M. De Stefano, F. Ungaro, M. I. La Rotonda, Cold field emission gun-scanning electron microscopy: a new tool for morphological and ultrastructural analysis of liposomes, Int. J. Pharm. 362 (2008) 189-192.

[40] K. Uekama, F. Hirayama, T. Irie, Cyclodextrin drug carrier systems, Chem. Rev. 98 (1998) 20452076.

[41] E. Nuin, M. Gomez-Mendoza, I. Andreu, M. L. Marin, M. A. Miranda, New photoactive compounds to probe cholic acid and cholesterol inside mixed micelles, Org. Lett. 15 (2013) 298301.

[42] I. Vaya, V. Lhiaubet-Vallet, M. C. Jimenez, M. A. Miranda, Photoactive assemblies of organic compounds and biomolecules: drug-protein supramolecular systems, Chem. Soc. Rev. 43 (2014) $4102-4122$.

[43] R. H. Muller, K. Mader, S. Gohla, Solid lipid nanoparticles (SLN) for controlled drug delivery - a review of the state of the art, Eur. J. Pharm. Biopharm. 50 (2000) 161-177. 
Chart 1<smiles>C[C@H](NCCCc1cccc(C(F)(F)F)c1)c1cccc2ccccc12</smiles><smiles>[X]N=[N+]=[N+]</smiles> 
Figure 1

A)

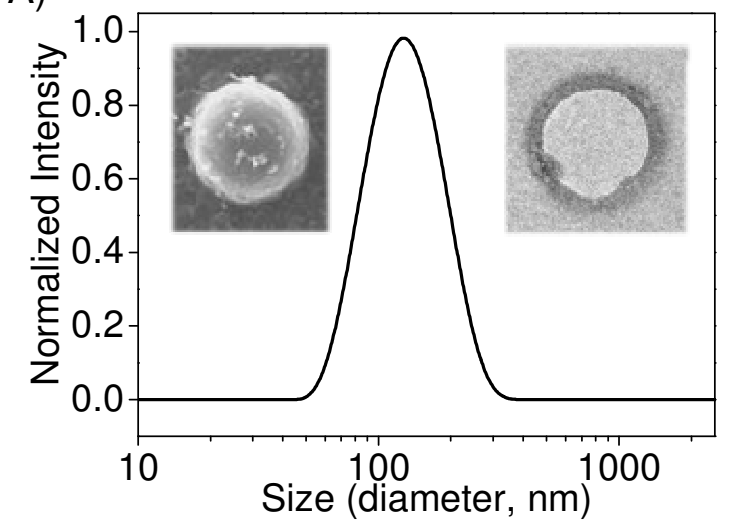

B)

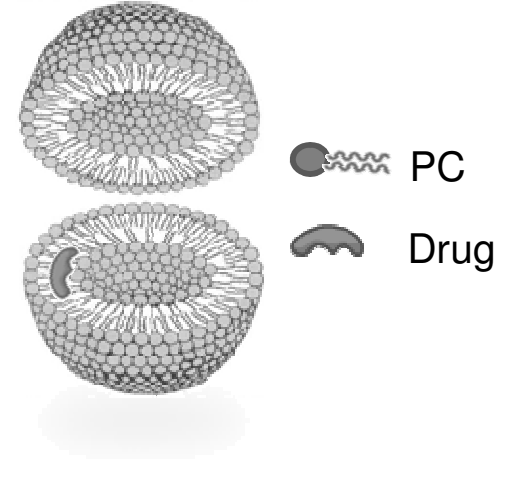


Figure 2

A)

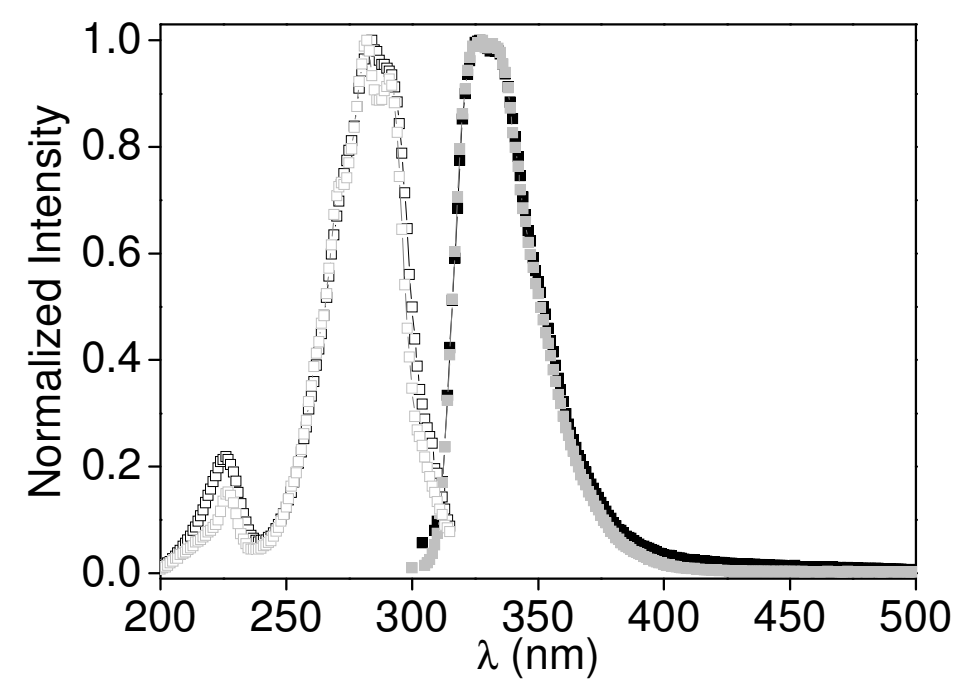

B)

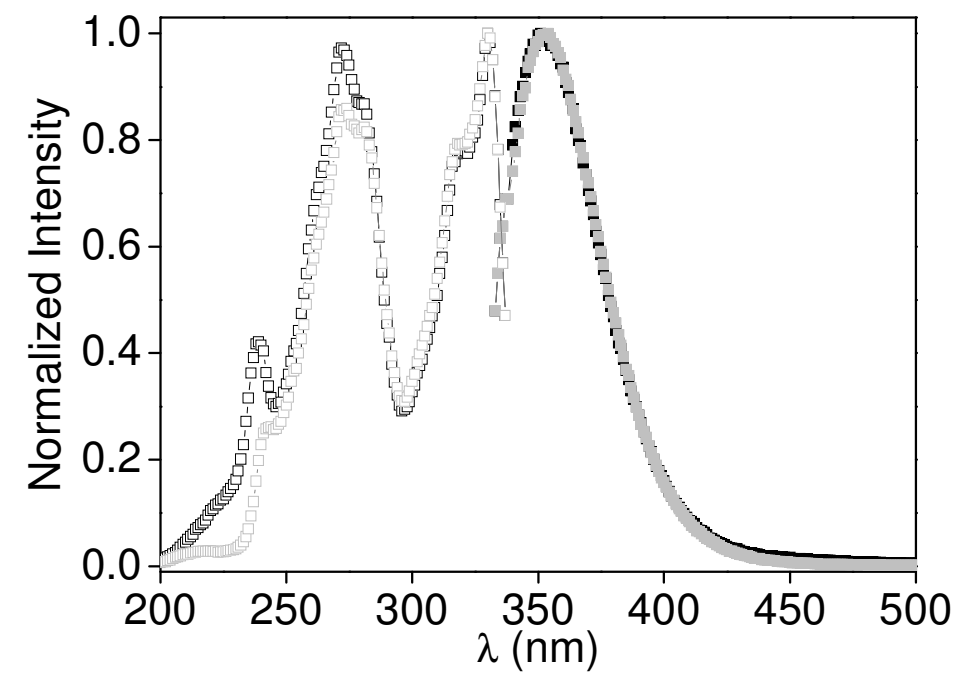


Figure 3

A)

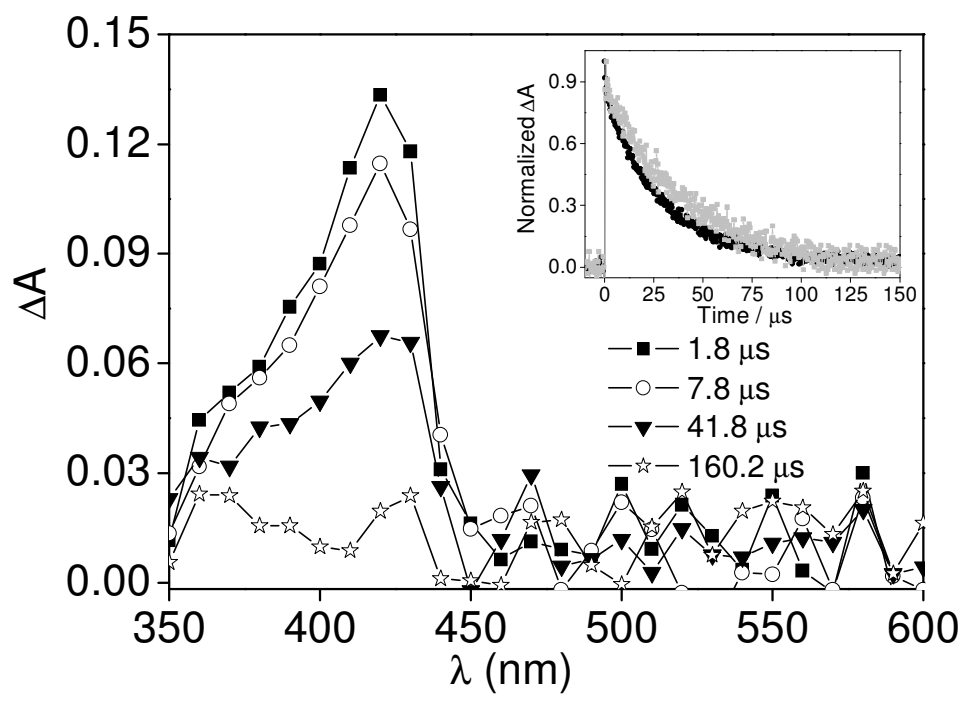

B)

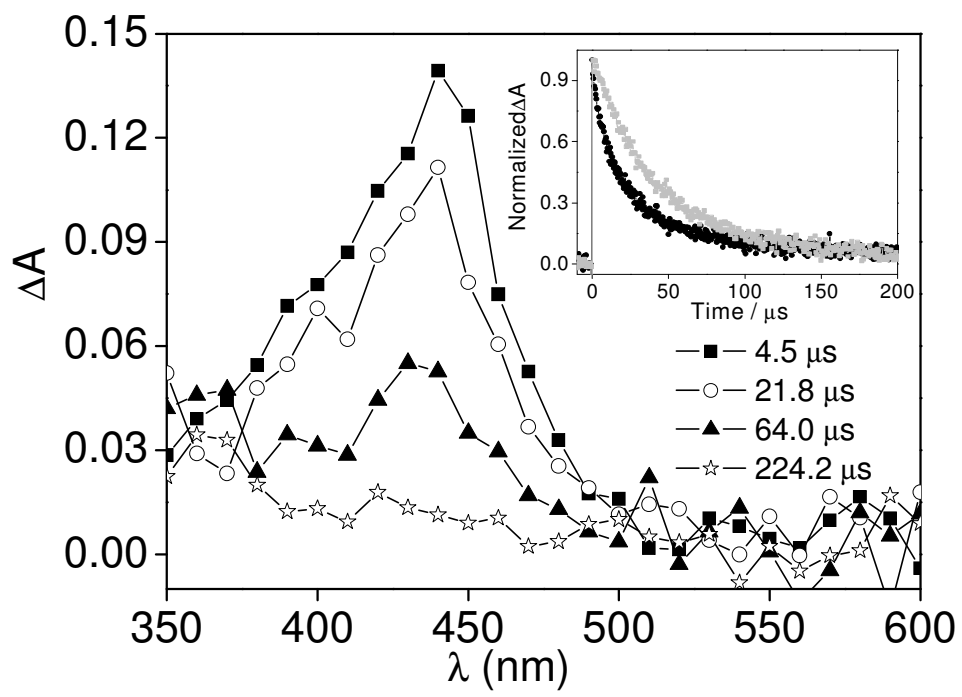


Table 1

\begin{tabular}{|c|c|c|c|c|c|}
\hline Sample & $\tau_{\mathbf{s}}(\mathbf{n s})^{\mathbf{a}}$ & $\mathbf{E}_{\mathbf{s}}(\mathbf{k c a l} / \mathbf{m o l})^{\mathbf{b}}$ & $\mathbf{k}_{\mathbf{F q}}\left(\mathbf{M}^{-\mathbf{1}} \mathbf{s e c}^{-\mathbf{1}}\right)^{\mathbf{c}}$ & $\tau_{\mathbf{T}}(\boldsymbol{\mu s})^{\mathbf{d}}$ & $\mathbf{k}_{\mathbf{T q}}\left(\mathbf{M}^{-\mathbf{1}} \mathbf{s e c}^{-\mathbf{1}}\right)^{\mathbf{e}}$ \\
\hline CIN & 31 & 92 & $1.4 \times 10^{10}$ & 29 & $1.8 \times 10^{9}$ \\
\hline CIN Lip & 30 & 92 & $1.5 \times 10^{10}$ & 46 & $1.2 \times 10^{9}$ \\
\hline NPX & 9.9 & 85 & $8.8 \times 10^{9}$ & 25 & $2.0 \times 10^{9}$ \\
\hline $\begin{array}{c}\text { NPX } \\
\text { Lip }\end{array}$ & 8.8 & 85 & $9.6 \times 10^{9}$ & 45 & $9.9 \times 10^{8}$ \\
\hline
\end{tabular}

${ }^{a}$ Singlet lifetime. ${ }^{b}$ Singlet energy. ${ }^{c}$ Fluorescence quenching rate constant for oxygen.

${ }^{\mathrm{d}}$ Triplet lifetime. ${ }^{\mathrm{e}}$ Triplet quenching rate constant for oxygen. 


\section{Captions}

Chart 1. Chemical structures of CIN and NPX

Figure 1. A) DLS size distribution curves for liposomes in PBS. Insets: CryoSEM image (left) and TEM image (right). B) Cartoon representation of the drug inside liposome.

Figure 2. Normalized emission ( $\mathbf{\square})$ and excitation $(\square)$ spectra of A) CIN ( $\lambda_{\text {exc }}=290 \mathrm{~nm}, \mathrm{~A}_{290}=0.1$ ) and B) NPX $\left(\lambda_{\text {exc }}=330 \mathrm{~nm}, \mathrm{~A}_{330}=0.1\right)$ in PBS solution (black) and inside liposome (grey).

Figure 3. Transient absorption spectra $\left(\lambda_{\text {exc }}=266 \mathrm{~nm}\right)$ under $\mathrm{N}_{2}$ of A) CIN and B) NPX inside liposomes. Insets: Normalized decay traces for the drugs in PBS (black) and within liposomes (grey) monitored at $420 \mathrm{~nm}$.

Table 1. Photophysical parameters of CIN and NPX outside and inside liposomes. 